\title{
Effects of Astragalus polysaccharides on memory impairment in a diabetic rat model
}

\author{
This article was published in the following Dove Press journal: \\ Neuropsychiatric Disease and Treatment \\ I July 2016 \\ Number of times this article has been viewed
}

\author{
Changping Dun' \\ Junqian Liu' \\ Fucheng Qiu' \\ Xueda $\mathrm{Wu}^{2}$ \\ Yakun Wang ${ }^{3}$ \\ Yongyan Zhao ${ }^{4}$ \\ Ping $\mathrm{Gu}^{\prime}$
}

'Department of Neurology, the First Hospital of Hebei Medical University, ${ }^{2}$ Department of Cardiac Surgery, the Second Hospital of Hebei Medical University, ${ }^{3}$ Department of Endocrinology, the Fourth Hospital of Hebei Medical University, Shijiazhuang, ${ }^{4}$ Department of Nursing, Maternal and Child Health Hospital of Tangshan City, Tangshan, People's Republic of China
Correspondence: Ping Gu

Department of Neurology, the First Hospital of Hebei Medical University, No 89 Donggang Road, Yuhua District, Shijiazhuang, 050030 Hebei, People's Republic of China

Tel/fax +86 31। I8633889939

Emailwjh19852008@I26.com
Objective: Astragalus polysaccharides (APS) are active constituents of Astragalus membranaceus. In this study, we aimed to investigate the effects of APS on memory impairment in a diabetic rat model and their mechanisms.

Methods: A diabetic model was established in 50 male Wistar rats with streptozotocin intraperitoneal injection. A blood glucose level higher than $16.7 \mathrm{mmol} / \mathrm{L}$ obtained 72 hours after the injection was regarded as a successful diabetic model. The modeled rats were divided into model group, high, medium, and low doses of APS, and piracetam groups (positive control). A group of ten rats without streptozotocin-induced diabetes were used as a normal control. After respective consecutive 8-week treatments, the levels of blood fasting plasma glucose, insulin, hemoglobin A1c, memory performance, hippocampal malondialdehyde, and superoxide dismutase were determined.

Results: After the 8-week APS treatment, serum fasting plasma glucose, hemoglobin A1c, and insulin levels were decreased compared with those of the model group $(P<0.05)$. Importantly, memory impairment in the diabetic model was reversed by APS treatments. In addition, hippocampal malondialdehyde concentration was lowered, whereas that of superoxide dismutase was higher after APS treatments.

Conclusion: APS are important active components responsible for memory improvement in rats with streptozotocin-induced diabetes. The potential mechanism of action is associated with the effects of APS on glucose and lipid metabolism, and antioxidative and insulin resistance. APS are constituents of $A$. membranaceus that are potential candidate therapeutic agents for the treatment of memory deficit in diabetes.

Keywords: diabetic, Astragalus polysaccharides, memory impairment, MDA, SOD

\section{Introduction}

Diabetes mellitus is the most common type of metabolic disorder worldwide, and its incidence is increasing annually. In 2014, it was estimated that $8.3 \%$ of adults globally have diabetes (International Diabetes Federation Atlas, 6th edition). ${ }^{1}$ Diabetes and insulin resistance are associated with changes in the central nervous system and the development of cognitive and memory impairments. The rising prevalence of diabetes and its earlier onset indicate that diabetes-related cognitive and memory dysfunction may increase substantially in the near future, causing tremendous socioeconomic burdens. Cognitive dysfunction affects the quality of life of diabetic patients and augments the risk of dementia., ${ }^{2,3}$ Therefore, the elucidation of the mechanisms of memory deficit and discovery of drugs with high therapeutic effects are urgently required in the treatment of diabetes.

The processes of learning and memory are related to the activities of a number of biological structures. The hippocampus is important for memory formation. ${ }^{4}$ Although 
the mechanisms underlying memory deficit in diabetes are not well understood, there are some implications from previous research. First, deviant sleep due to impaired glucose metabolism is shown to produce memory deficits in patients with diabetes mellitus. ${ }^{5}$ Second, chronic hyperglycemia in rats was reported to result in a significant loss of cortical neurons, which may impair cognition. ${ }^{6}$ In addition, neurotransmitter systems, such as gamma-aminobutyric acid, and cholinergic systems involved in learning and memory are affected. ${ }^{7}$ The findings of animal studies also indicate that chronic hyperglycemia induced by streptozotocin (STZ) decreases the synthesis and release of acetylcholine in rat brain. ${ }^{8}$

Herbal medicines have long been an attractive option in the treatment of diabetes. Astragalus mongholicus (Huangqi) was documented to reduce blood glucose level in diabetic patients. $^{9-11}$ More importantly, it has the ability to ameliorate brain functions in a diabetic model. ${ }^{12}$ However, the exact ingredients involved in the effect of $A$. mongholicus are not identified. The active constituents of the plant include polysaccharides, saponins, flavonoids, L-arginine, and L-canavanine. ${ }^{9,13}$ Among these, Astragalus polysaccharides (APS) have been most widely investigated, mainly with respect to their immunopotentiating properties, ability to counteract the side effects of chemotherapeutic drugs, and anticancer activities. ${ }^{13-15}$ In this study, APS were applied in a STZ-induced diabetic rat model to explore their potential protective mechanisms.

\section{Materials and methods Diabetic model and treatment}

Fifty-four SPF-grade male Wistar rats (weighing 260-380 g, Application No: 20140038) were obtained from the Animal Center of Shandong University and housed in a 12-hour light-dark cycle with food and water ad libitum under controlled temperature $\left(23^{\circ} \mathrm{C} \pm 2^{\circ} \mathrm{C}\right)$ and humidity $(55 \% \pm 5 \%)$. The animal experiments were reviewed and approved by the Animal Care and Use Committee of the First Hospital of Hebei Medical University and performed in accordance with the Guidelines for the Care and Use of Laboratory Animals. All were subjected to fasting for 16 hours. Then, 50 of them were intraperitoneally injected once with $10 \%$ $\mathrm{STZ}(50 \mathrm{mg} / \mathrm{kg})$. STZ was dissolved in citrate buffer with $\mathrm{pH}$ adjusted to a value of 4.5. Seventy-two hours after injection, blood was drawn from the tail vein, and blood glucose levels were determined. The diabetic models were regarded as successful if their blood glucose levels were higher than $16.7 \mathrm{mmol} / \mathrm{L}$. The animals with diabetes were divided randomly into five groups $(\mathrm{n}=9 /$ group): model group, piracetam group, group treated with a high-dose of APS ( $800 \mathrm{mg} / \mathrm{kg} /$ day), group treated with a medium-dose of APS (400 mg/kg/day), and group treated with a low-dose of APS (200 mg/kg/day). APS and piracetam were administered orally for 8 consecutive weeks. Piracetam (Batch No: H12020667, Tianjing Jinshi Pharmacy Co. Ltd., Tianjin, People's Republic of China) was applied as a positive control at a dose of $500 \mathrm{mg} / \mathrm{kg} /$ day. APS (Xi'an Ruilin Biotech, Xi'an, People's Republic of China) were prepared as suspension liquid. A group of ten rats without STZ injection was set as a normal control.

\section{FPG, $\mathrm{HbAlc}$, and insulin measurements}

The levels of serum blood glucose were measured every 4 weeks. Twenty-four hours after the last drug treatment, vein blood was collected for fasting plasma glucose (FPG), hemoglobin A1c (HbA1c), and insulin measurements (Sigma-Aldrich Co., St Louis, MO, USA).

\section{Morris water maze}

Animals were tested by Morris water maze 8 weeks after the treatments. At the beginning of the place navigation task, rats were placed in a pool without a platform, allowing them to swim for 5 minutes and familiarize with the environment. Tests were carried out at a fixed time every day for 5 days, and there were four training sections during each time period (120 seconds). Rats were placed into the pool facing the wall at any of the four starting points. Video camera tracking system recorded the time necessary for rats to find the platforms (escape latency) and their swimming routes. During the four training sections, rats were placed into the pool at four different starting points (different quadrants). If the animals did not find the platforms within 120 seconds during the Morris water maze test, the latency was assigned 120 seconds. Then, the rats were positioned onto the platforms, allowing a 15 second rest before the next training section. The average value of latency during four training sections performed every day was recorded as the study result of that day. On the sixth day of spatial probe, the platform was removed, and rats were placed into water at a randomly selected, but the same for all animals, starting point. The time interval during which rats stayed in the quadrant with the platform was recorded.

\section{MDA and SOD measurements}

After the behavioral measurements, the rats were decapitated. Hippocampus was isolated for malondialdehyde (MDA) and superoxide dismutase (SOD) tests. Hippocampal 
MDA and SOD activities were detected by assay kits (Sigma-Aldrich Co.).

\section{Statistical analysis}

All data were presented as mean \pm standard deviation. Differences among three or more groups were compared by one-way analysis of variance, followed by Bonferroni post hoc testing for multiple comparisons. Differences between two groups were assessed by the Student's $t$-test. $P$-values of 0.05 or less were regarded as significant.

\section{Results}

\section{APS decreased FPG and HbAIc levels in the diabetic model}

As compared with the normal control, the induction of diabetes resulted in elevated blood FPG and HbA1c levels (Table 1). After treatments for 8 weeks, FPG level was significantly decreased in the groups with the three doses of APS and the piracetam group ( $P<0.05$ vs model).

HbA1c level was also increased in the diabetic model group compared with the normal control. APS or piracetam treatments attenuated the elevated $\mathrm{HbAlc}$ concentrations in the diabetic model $(P<0.05$ vs model) (Table 1$)$.

\section{APS reduced serum insulin level}

After diabetic modeling, the insulin level was considerably downregulated when compared with the normal control. The treatment with APS at all three doses or with piracetam significantly elevated the insulin level $(P<0.05$ vs model $)$ (Table 2).

\section{APS ameliorated the memory impairment in the diabetic model}

Compared with the normal control, the latency to find the platform was significantly increased in the diabetic model group $(P<0.05)$. However, the treatment with APS at all three doses or with piracetam restored the latency $(P<0.05$ vs model) (Table 3). On the sixth day, after removal of the platform, the rats in the diabetic model group spent less time in the target quadrant compared with the normal control $(P<0.05$ vs control). However, the treatment with APS at all three doses or with piracetam apparently restored the memory deficit ( $P<0.05$ vs model) (Table 4$)$.

\section{APS elevated SOD and MDA levels in the diabetic model}

After modeling, hippocampal SOD activity was significantly decreased, whereas MDA level was increased. After the treatments, the SOD concentration was substantially augmented in the treatments with the three doses of APS and with piracetam. By contrast, the MDA level was decreased after the treatments with APS or piracetam $(P<0.05$ vs model) (Table 5).

\section{Discussion}

Huangqi is a common herbal medicine widely applied in different prescriptions for therapy of diseases, especially in Korea and People's Republic of China. As documented, Huangqi possesses antioxidative and cardiovascular ameliorating activities. ${ }^{16,17}$ The herb has been utilized to treat cerebral ischemia ${ }^{18,19}$ and maintain the integrity of the blood-brain barrier. ${ }^{20}$ Its application was also effective in the therapy of diabetic nephropathy, and by ameliorating the function of kidneys, it caused a reduction in urine protein excretion. ${ }^{21,22}$ Clinically, Huangqi was reported to reverse memory impairment in diabetic patients. ${ }^{23-25}$ These findings indicate the cerebral protection promoted by the drug. Mechanically, it is likely that Huangqi exerts its neuroprotective action through its anti-inflammatory, antioxidative, and antiapoptotic activities. ${ }^{26}$ Nevertheless, the exact ingredients

Table I FPG and HbAIc levels in different rat groups (mean $\pm \mathrm{SD}, \mathrm{n}=\mathrm{I0}$ )

\begin{tabular}{|c|c|c|c|c|}
\hline \multirow[t]{2}{*}{ Groups } & \multicolumn{3}{|c|}{ FPG (mmol/L) } & \multirow[t]{2}{*}{ HbAlc (\%) } \\
\hline & $0 \mathrm{w}$ & $4 w$ & $8 w$ & \\
\hline Control & $6.66 \pm 0.19$ & $6.77 \pm 0.26$ & $6.90 \pm 0.49$ & $4.55 \pm I .4 I$ \\
\hline DM & $20.78 \pm 2.22 *$ & $21.61 \pm 2.21 *$ & $20.11 \pm 1.22^{*}$ & $9.13 \pm 1.12 *$ \\
\hline Piracetam & $21.69 \pm 2.5 I^{*}$ & $|8.33 \pm| .09 *$ & $16.69 \pm 0.89 *$ & $7.12 \pm 1.09 *$ \\
\hline Low dose of APS & $21.68 \pm 3.07 *$ & $19.89 \pm 2.09 *$ & $17.33 \pm 2.48^{*, \square, \Delta}$ & $8.56 \pm 1.44^{*, \square \Delta}$ \\
\hline Medium dose of APS & $23.5 I \pm 4.88^{*}$ & $20.69 \pm 2.89 *$ & $16.87 \pm 1.57^{*, \square, \Delta}$ & $7.78 \pm|.2|^{*, \square, \Delta}$ \\
\hline High dose of APS & $24.56 \pm 5.12 *$ & $20.22 \pm 2.45 *$ & $16.13 \pm 2.22^{* \square, \Delta}$ & $7.23 \pm 1.15^{*, \square \Delta}$ \\
\hline
\end{tabular}

Notes: $* P=0.0014<0.05$ compared with the control; $\square=0.0000<0.05$ compared with the model group; ${ }^{\triangle} P=0.0000<0.05$ compared with the piracetam group.

Abbreviations: APS, Astragalus polysaccharides; DM, diabetes mellitus; FPG, fasting plasma glucose; HbAlc, glycated hemoglobin; SD, standard deviation; w, weeks. 
Table 2 Serum insulin levels in different groups (mean $\pm S D$, $\mathrm{n}=10$ )

\begin{tabular}{ll}
\hline Groups & Insulin level/(ulU/mL) \\
\hline Control & $17.23 \pm 7.32$ \\
DM & $12.03 \pm 2.39 *$ \\
Piracetam & $14.44 \pm 5.29 *, \square$ \\
Low dose of APS & $17.67 \pm 6.56^{*, \square, \Delta}$ \\
Medium dose of APS & $16.55 \pm 6.78^{*, \square, \Delta}$ \\
High dose of APS & $14.22 \pm 2.19^{*, \square, \Delta}$ \\
\hline
\end{tabular}

Notes: $* P=0.00 \mid 4<0.05$ compared with the control; $\square=0.0000<0.05$ compared with the model group; ${ }^{\triangle P}=0.0000<0.05$ compared with the piracetam group.

Abbreviations: APS, Astragalus polysaccharides; DM, diabetes mellitus; SD, standard deviation.

responsible for the protection are not identified. In this study, we evidenced that APS, constituting one of the major components, ameliorated the memory impairment in diabetic model rats. These data implicate the action of these compounds for inducing the neuroprotective effects of the herb.

APS are the most important components in Astragalus membranaceus and have been clinically applied in the treatment of cancer as an adjunctive medicine enhancing immune activity. ${ }^{15}$ APS are also effective for the treatment of diabetic nephropathy, possibly through ameliorating glucose metabolism and decreasing the levels of transform growth factor beta $1 .{ }^{27}$ In addition, APS are involved in oxidative stress elimination and insulin resistance..$^{28}$ Based upon this evidence, APS present a high potential to reverse memory deficit in diabetic animal models.

In our study, diabetic rats underwent 8-week APS treatment. The latency to find the platform was significantly shortened after the treatment. Meanwhile, serum FPG and $\mathrm{HbAlc}$ levels were remarkably decreased. These data implicate that glucose metabolism is altered after a treatment with APS. APS also decreased the insulin level, indicating the influence of APS on insulin resistance. In addition, we

Table 3 Latencies to find the platform in different groups (mean \pm $\mathrm{SD}, \mathrm{n}=10$ )

\begin{tabular}{lll}
\hline Groups & Latency (s) \\
\cline { 2 - 3 } & Before treatment & Posttreatment \\
\hline Control & $45.44 \pm 2.45$ & $47.21 \pm 2.33$ \\
DM & $83.11 \pm 5.13^{*}$ & $86.35 \pm 4.77^{*}, \square$ \\
Piracetam & $74.32 \pm 4.34^{*}$ & $44.23 \pm 4.54^{*}$, \\
Low dose of APS & $71.11 \pm 2.37^{*}$ & $43.66 \pm 4.55^{*, \square}$ \\
Medium dose of APS & $73.67 \pm 4.24^{*}$ & $42.45 \pm 4.23^{*} \square$ \\
High dose of APS & $70.56 \pm 3.33^{*}$ & $40.34 \pm 3.2 I^{*} \square$ \\
\hline
\end{tabular}

Notes: $* P=0.0014<0.05$ compared with the control; $\square=0.0000<0.05$ compared with the model group.

Abbreviations: APS, Astragalus polysaccharides; DM, diabetes mellitus; SD, standard deviation.
Table 4 The time spent in the target quadrant by the different groups (mean $\pm S D, n=10$ )

\begin{tabular}{lll}
\hline Groups & Before treatment (\%) & Posttreatment (\%) \\
\hline Control & $38.8 \pm 1.4$ & $37.3 \pm 1.9$ \\
DM & $20.6 \pm 1.3^{*}$ & $21.2 \pm 2.1^{*, \square}$ \\
Piracetam & $20.3 \pm 1.2^{*}$ & $33.4 \pm 3.7^{*, \square}$ \\
Low dose of APS & $20.8 \pm 1.3^{*}$ & $31.2 \pm 1.8^{*, \square}$ \\
Medium dose of APS & $20.1 \pm 1.5^{*}$ & $28.2 \pm 2.8^{*, \square}$ \\
High dose of APS & $20.2 \pm 1.3^{*}$ & $34.3 \pm 2.7^{*, \square}$ \\
\hline
\end{tabular}

Notes: $* P=0.0014<0.05$ compared with the control; $\square=0.0000<0.05$ compared with the model group.

Abbreviations: APS, Astragalus polysaccharides; DM, diabetes mellitus; SD, standard deviation.

measured the hippocampal MDA and SOD levels. MDA is an important biomarker of lipid metabolism. ${ }^{29}$ The decrease of MDA after the APS treatment indicated that the regulation of the lipid metabolism of the experimental animals was improved after the APS treatment. SOD is a marker of antioxidative ability. ${ }^{30}$ The increase in SOD concentrations revealed that the antioxidative ability was increased after the application of APS.

We explored the impact of APS treatment on the recovery of memory and its underlying mechanisms. However, there are a large number of other bioactive components present in the herb. More clinical studies are required to classify the active components involved in the neuroprotective activities of A. membranaceus in diabetes.

\section{Conclusion}

Through this study, we provide evidence that APS are important active components responsible for the improvement of memory impairment caused by diabetes. The potential mechanism of action is associated with the effects of APS on glucose and lipid metabolism, their antioxidative properties, and influence on insulin resistance. APS are potential candidate therapeutic agents for the treatment of memory deficit in diabetes.

Table 5 Hippocampal SOD and MDA levels in the different groups (mean $\pm \mathrm{SD}, \mathrm{n}=10$ )

\begin{tabular}{lll}
\hline Groups & $\begin{array}{l}\text { SOD (U/mg } \\
\text { protein) }\end{array}$ & $\begin{array}{l}\text { MDA (mmol/mg } \\
\text { protein) }\end{array}$ \\
\hline Control & $10.22 \pm 0.19$ & $4.77 \pm 1.45$ \\
DM & $6.67 \pm 0.29^{*}$ & $12.56 \pm 1.15^{*}$ \\
Piracetam & $7.78 \pm 0.23^{*, \square}$ & $9.33 \pm 0.73^{*, \square}$ \\
Low dose of APS & $6.65 \pm 0.33^{*, \square, \Delta}$ & $11.41 \pm 1.47^{*, \square, \Delta}$ \\
Medium dose of APS & $7.13 \pm 0.30^{*, \square, \Delta}$ & $10.45 \pm 1.09^{*, \square, \Delta}$ \\
High dose of APS & $8.22 \pm 0.31^{*, \square, \Delta}$ & $10.41 \pm 0.48^{*, \square, \Delta}$ \\
\hline
\end{tabular}

Notes: $* P=0.0014<0.05$ compared with the control; $\square=0.0000<0.05$ compared with the model group; $\triangle P=0.0000<0.05$ compared with the piracetam group.

Abbreviations: APS, Astragalus polysaccharides; DM, diabetes mellitus; MDA, malondialdehyde; SD, standard deviation; SOD, superoxide dismutase. 


\section{Disclosure}

The authors report no conflicts of interest in this work.

\section{References}

1. Kirtland KA, Cho P, Geiss LS. Diabetes Among Asians and Native Hawaiians or other Pacific Islanders-United States, 2011-2014. MMWR Morb Mortal Wkly Rep. 2015;64(45):1261-1266.

2. Elias PK, Elias MF, D'Agostino RB, et al. NIDDM and blood pressure as risk factors for poor cognitive performance. The Framingham Study. Diabetes Care. 1997;20(9):1388-1395.

3. Ott A, Stolk RP, Hofman A, van Harskamp F, Grobbee DE, Breteler MM. Association of diabetes mellitus and dementia: the Rotterdam Study. Diabetologia. 1996;39(11):1392-1397.

4. Hasselmo ME, Wyble BP, Wallenstein GV. Encoding and retrieval of episodic memories: role of cholinergic and GABAergic modulation in the hippocampus. Hippocampus. 1996;6(6):693-708.

5. Herzog N, Friedrich A, Fujita N, et al. Effects of daytime food intake on memory consolidation during sleep or sleep deprivation. PLoS One. 2012;7(6):e40298.

6. Redish AD, Touretzky DS. Cognitive maps beyond the hippocampus. Hippocampus. 1997;7(1):15-35.

7. Everitt BJ, Robbins TW. Central cholinergic systems and cognition. Annu Rev Psychol. 1997;48:649-684.

8. Welsh B, Wecker L. Effects of streptozotocin-induced diabetes on acetylcholine metabolism in rat brain. Neurochem Res. 1991;16(4): 453-460

9. Zhang K, Pugliese M, Pugliese A, Passantino A. Biological active ingredients of traditional Chinese herb Astragalus membranaceus on treatment of diabetes: a systematic review. Mini Rev Med Chem. 2015; 15(4):315-329.

10. Xin MU, Yuan X, Kang B, Guo rong HE, Guan hua DU. The mRNA expression of renal aquaporin-2 in rats with diabetes mellitus and the therapeutical effect of Astragalus membranaceus. Chinese Pharmacol Bull. 2010;26(9):1176-1179.

11. Zhao H, Wu Y, Huang S. Screening of differentially displayed genes correlative to diabetes with and without Chinese herb Astragalus membranaceus treatment. Faseb J. 2006;20(5):LB71.

12. Li M, Wang W, Xue J, Gu Y, Lin S. Meta-analysis of the clinical value of Astragalus membranaceus in diabetic nephropathy. J Ethnopharmacol. 2011;133(2):412-419.

13. Hou YC, Wu JM, Wang MY, et al. Modulatory effects of Astragalus polysaccharides on T-cell polarization in mice with polymicrobial sepsis. Mediators Inflamm. 2015;2015:826319.

14. Li R, Chen WC, Wang WP, Tian WY, Zhang XG. Extraction, characterization of Astragalus polysaccharides and its immune modulating activities in rats with gastric cancer. Carbohydr Polym. 2009; 78(4):738-742.

15. Guo L, Bai SP, Zhao L, Wang XH. Astragalus polysaccharide injection integrated with vinorelbine and cisplatin for patients with advanced non-small cell lung cancer: effects on quality of life and survival. Med Oncol. 2012;29(3):1656-1662.

Neuropsychiatric Disease and Treatment

\section{Publish your work in this journal}

Neuropsychiatric Disease and Treatment is an international, peerreviewed journal of clinical therapeutics and pharmacology focusing on concise rapid reporting of clinical or pre-clinical studies on a range of neuropsychiatric and neurological disorders. This journal is indexed on PubMed Central, the 'PsycINFO' database and CAS,
16. Shahzad M, Small DM, Morais C, Wojcikowski K, Shabbir A, Gobe GC. Protection against oxidative stress-induced apoptosis in kidney epithelium by Angelica and Astragalus. J Ethnopharmacol. 2016; 179:412-419.

17. Pu W, Wang D, Zhou D. Structural characterization and evaluation of the antioxidant activity of phenolic compounds from Astragalus taipaishanensis and their structure-activity relationship. Sci Rep. 2015; 5:13914.

18. Jia RZ, Jiang L, Qiao LX. 黄芪对新生鼠缺氧缺血脑损伤后皮质的治 疗作用 [Study on effect of radix astragali on injury of cerebral cortex in neonatal rats after hypoxia/ischemia brain damage]. Zhongguo Zhong Xi Yi Jie He Za Zhi. 2005;25(1):54-57. Chinese.

19. Quan J, Du G. [Protective effect of Astragalus membranaceus (Fisch.) Bge. and Hedysarum polybotrys Hand.-Mazz. on experimental model of cerebral ischemia in rats]. Zhongguo Zhong Yao Za Zhi. 1998; 23(6):371-373. Chinese.

20. Cai J, Pan R, Jia X, et al. The combination of astragalus membranaceus and ligustrazine ameliorates micro-haemorrhage by maintaining bloodbrain barrier integrity in cerebrally ischaemic rats. $J$ Ethnopharmacol. 2014;158:301-309.

21. Kim J, Moon E, Kwon S. Effect of Astragalus membranaceus extract on diabetic nephropathy. Endocrinol Diabetes Metab Case Rep. 2014; 2014:140063.

22. Ji L, Chen X, Zhong X, et al. Astragalus membranaceus up-regulate Cosmc expression and reverse IgA dys-glycosylation in IgA nephropathy. BMC Complement Altern Med. 2014;14:195.

23. Tohda C, Tamura T, Matsuyama S, Komatsu K. Promotion of axonal maturation and prevention of memory loss in mice by extracts of Astragalus mongholicus. Br J Pharmacol. 2006;149(5):532-541.

24. Hong GX, Qin WC, Huang LS. [Memory-improving effect of aqueous extract of Astragalus membranaceus (Fisch.) Bge]. Zhongguo Zhong Yao Za Zhi. 1994;19(11):687-688. Chinese.

25. Liu ZY, Yang YG, Zheng B. [Effect of improving memory and inhibiting acetylcholinesterase activity by invigorating-qi and warming-yang recipe]. Zhongguo Zhong Xi Yi Jie He Za Zhi. 1993;13(11):675-676. Chinese.

26. Li X, Qu L, Dong Y, et al. A review of recent research progress on the astragalus genus. Molecules. 2014;19(11):18850-18880.

27. Zhang YW, Wu CY, Cheng JT. Merit of Astragalus polysaccharide in the improvement of early diabetic nephropathy with an effect on mRNA expressions of NF-kappaB and IkappaB in renal cortex of streptozotoxin-induced diabetic rats. J Ethnopharmacol. 2007;114(3):387-392.

28. Liu M, Wu K, Mao X, Wu Y, Ouyang J. Astragalus polysaccharide improves insulin sensitivity in KKAy mice: regulation of PKB/GLUT4 signaling in skeletal muscle. J Ethnopharmacol. 2010;127(1):32-37.

29. Niki E. Biomarkers of lipid peroxidation in clinical material. Biochim Biophys Acta. 2014;1840(2):809-817.

30. Tiwari BK, Pandey KB, Abidi AB, Rizvi SI. Markers of oxidative stress during diabetes mellitus. J Biomark. 2013;2013:378790.

\section{Dovepress}

and is the official journal of The International Neuropsychiatric Association (INA). The manuscript management system is completely online and includes a very quick and fair peer-review system, which is all easy to use. Visit http://www.dovepress.com/testimonials.php to read real quotes from published authors. 